\title{
Segurança do doente e os processos sociais na relação com enfermeiros em contexto de bloco operatório
}

\section{La seguridad del enfermo y los procesos sociales en relación con enfermeros en el contexto de la sala de operaciones}

\section{Patient safety and social processes in the relationship with nurses in the operating room context}

Edite Lopes da Silva ${ }^{1}$ y Francisco Rafael de Araújo Rodrigues ${ }^{2}$

${ }^{1}$ Enfermera. Master. Estudiante de doctorado en Ciencias de la Enfermería en la Universidad del Porto. Portugal. Profesor Asistente de enfermería de la Universidad del Cabo Verde. Cabo Verde.

${ }^{2}$ Enfermero. Master. Estudiante de doctorado en Ciencias de la Enfermería en la Universidad do Porto. Portugal.

Cómo citar este artículo en edición digital: Silva, E.L., y Araujo Rodríguez, F.R. (2016). La seguridad del enfermo y los procesos sociales en relación con enfermeros en el contexto de la sala de operaciones

Cultura de los Cuidados (Edición digital), 20(46). Disponible en: < http://dx.doi.org/10.14198/cuid.2016.46.13>

Correspondência: Francisco Rafael de Araújo Rodrigues. Calle D. Pedro V, no 223 apto: - 204 F/CP: 4150-603.

Massarelos, Porto - Portugal.

Correo electrónico: rafaelrodrigues.rfl@gmail.com

Recibido: 30/09/2015; Aceptado: 23/07/2016

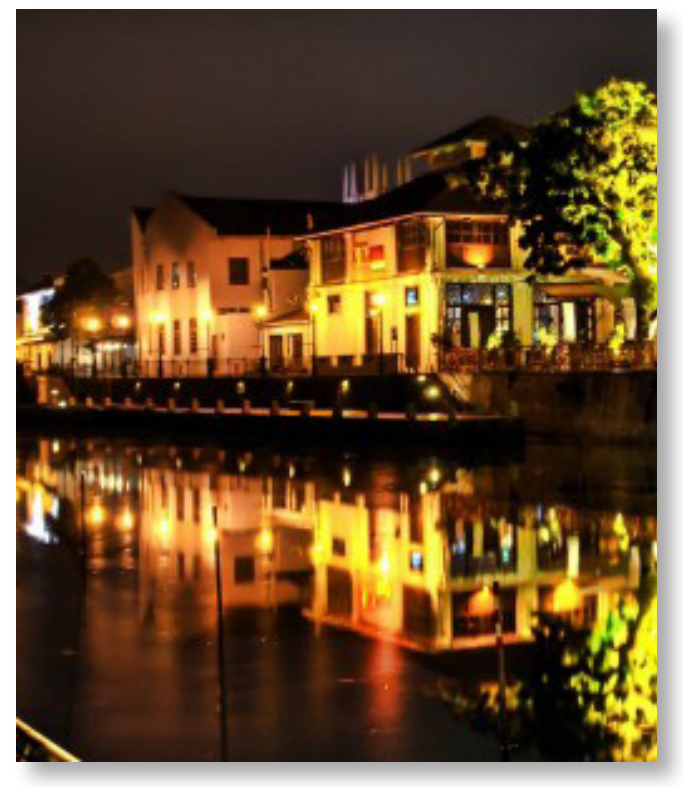

ABSTRACT

Patient safety is a worldwide problem that affects countries in all levels of development, given the risk of damages, injuries or death due to unsafe health care provision in the surgical context. This study aimed to understand the perception of nurses about patient safety in the operating room context. Descriptive and exploratory study conducted in $50 \mathrm{mo-}$ ments of participant observation and 6 semistructured interviews with nurses from the central operating room in a Public Hospital of the Sotavento Islands, Praia, Cape Verde. For data interpretation, we applied content analysis techniques. The ethical principles were followed. The explanatory cultural identity of the social processes in that context revealed unfavorable relation to patient safety as regards the aspects of surgical hand scrubbing with 2to 5-minute scrub with chlorhexidine, use of surgical safety checklist, verifying sterilization indicators, conflict management and communication among the interdisciplinary team, people as centrality of care. Both professionals in the operating room and institutions should consider standards, supervisions, audits, and protocols as strong points for patient safety in the operating room context.

Keywords: Patient Safety; Surgical Safety; Operating Room, Nursing Care. 


\section{RESUMEN}

La seguridad del paciente es un problema global que afecta a los países en todos los niveles de desarrollo, debido a riesgo de daños, lesiones o muerte por no provisión segura de la atención de salud en el ámbito quirúrgico. El objetivo fue comprender la percepción de enfermeros sobre seguridad del paciente en el contexto de la sala de operaciones. Estudio descriptivo, en 50 momentos de la observación participante y seis entrevistas semiestructuradas con enfermeros de la sala de operaciones central en un hospital público de las Islas de Sotavento, Praia, en Cabo Verde. La interpretación se basa en técnicas de análisis de contenido. Se respetaron los principios éticos. $\mathrm{La}$ identidad cultural explicativa de los procesos sociales en aquello contexto señaló relación desfavorable para seguridad del paciente, en los aspectos lavado de manos quirúrgico con copelación durante 2-5 minutos con clorhexidina, uso de lista de comprobación para cirugía segura, verificación de indicadores de esterilización, gestión de conflictos y comunicación entre equipo interdisciplinario, persona como centralidad de la atención. Profesionales del quirófano y las instituciones deben considerar normas, supervisión, auditoría y protocolos como puntos fuertes para seguridad del paciente en el contexto de la sala de operaciones.

Palabras clave: seguridad del paciente, seguridad quirúrgica, sala de operaciones, atención de enfermería.

\section{RESUMO}

A segurança do doente é um problema global que afeta países em todos os níveis de desenvolvimento, visto os riscos de danos, lesões ou morte devido à prestação de cuidados de saúde insegura em contexto cirúrgico. Objeti- vou-se compreender a percepção de enfermeiros sobre a segurança do doente no contexto do bloco operatório. Estudo descritivo e exploratório, realizado em 50 momentos de observação participante e seis entrevistas semiestruturadas com enfermeiros do bloco operatório central, em um Hospital Público das ilhas de Sotavento, Cidade da Praia, em Cabo-Verde. A interpretação fundamentou-se nas técnicas de análise de conteúdo. Os preceitos éticos foram respeitados. A identidade cultural explicativa dos processos sociais naquele contexto evidenciou relação não favorável para a segurança do doente, nos aspectos lavagem cirúrgica das mãos com escovação durante 2 a 5 minutos, com solução de clorexidina, utilização de checklist para cirurgias seguras, verificação de indicadores de esterilização, gestão de conflitos e comunicação entre a equipe interdisciplinar, pessoa como centralidade dos cuidados. Os profissionais do bloco operatório e as instituições devem considerar normas, supervisões, auditorias e protocolos como pontos fortes para segurança do doente no contexto do bloco operatório.

Palavras-chave: segurança do paciente, segurança cirúrgica, bloco operatório, cuidados de enfermagem.

\section{INTRODUÇÃO}

A Organização Mundial da Saúde (OMS) recomenda a redução de riscos nos cuidados de saúde (WHO, 2009) e a promoção de um ambiente seguro tanto para os doentes quanto para os profissionais (Cambotas, 2015). Os serviços de saúde ao adotarem, nas práticas, as recomendações da OMS, incorporam metodologias de gestão de risco e segurança como indicadores específicos para contínua qualidade dos cuidados prestados (Alpendre, 2014). 
Os eventos ou circunstâncias que possam vir acontecer na prestação de cuidados resultado de complicações ou danos à saúde da pessoa, afetam, em média, 10\% dos doentes admitidos em hospitais. O erro humano assume um dos antecedentes que causa grande impacto na sociedade e acarreta consequências éticos e legais aos profissionais de saúde (Duarte, Stipp, Silva, \& Oliveira, 2015).

A síntese teórica que se estrutura no contexto internacional aponta que um em cada seis doentes internados em instituições hospitalares esteve em condição de vulnerabilidade para algum tipo de erro ou evento adverso, mesmo sendo possível realização de estratégias preventivas (Paranaguá, Braga, Bezerra, Camargo-Silva, Azevedo-Filho, \& Sousa, 2014). O relatório do Institute of Medicine (IOM), dos Estados Unidos da América, apresentou que dos 33,6 milhões dos doentes internados, cerca de 44.000 a 98.000 americanos morreram em um ano, em consequência dos eventos adversos (Pereira, Souza, \& Ferraz, 2014).

Para a OMS (Gebrim, Melchior, Amaral, Barreto, \& Palos, 2014), estima-se que mundialmente cerca de 234 milhões de cirurgias são realizadas a cada ano, destas, aproximadamente 7 milhões estão sujeitas às complicações cirúrgicas, sendo que cerca de 1 milhão com casos de morte no intra-operatório e pós-operatório, e 50\% são preveníveis.

Desse modo, o olhar interdisciplinar da equipe multiprofissional em saúde é necessário para os cuidados congruentes e eficazes. Rodrigues (2015:15) explica que:

O processo de cuidar requer uma abordagem de interdisciplinar que deve ser desenvolvida num reconhecimento de competências de cada profissão, numa atitude dialógica e reflexiva, onde o confronto das ideias e os conhecimentos de todos os sujeitos envolvidos são le- vados em consideração para a (re) construção de novos conceitos, superação de intervenções descontextualizadas e padronizadas e, exploração do saber definidos.

A enfermagem constitui-se indispensável ao trabalho em equipa do bloco operatório, por congregar competências técnico-científicas e gerenciais necessárias para preparação de doentes, estabelecendo e desenvolvendo cuidados de enfermagem, de acordo com a especificidade da cirurgia. Para Chistóforo, Zagonel, \& Carvalho (2006), estes cuidados incluem desde a preparação física e emocional, orientação e avaliação ao encaminhamento do doente à sala cirúrgica, no sentido de minimizar as complicações pós-cirúrgicas.

Diante da fundamentação explanada, questionou-se: quais as características relacionadas à segurança do doente a partir da ótica de enfermeiros, no contexto do bloco operatório?

Logo, este estudo objetivou compreender a percepção de enfermeiros sobre a segurança do doente no bloco operatório. E, especificamente objetivou descrever as características que se manifestam mais adequadas para a segurança do doente; analisar aspectos cognitivos e comportamentais expressos por enfermeiros no processo de cuidar seguro. Identificar a construção identitária assumida por enfermeiros, a partir das vivências em uma relação interdisciplinar que remetem ao processo de cuidado seguro.

\section{METODOLOGIA}

\section{Desenho do estudo}

O desenho apoia-se no paradigma construtivista, em uma validação indutiva dos resultados que são produto de uma realidade social (Corbin \& Strauss, 2008) e em função dos objetivos, portanto, o estudo se classifica como descritivo e exploratório. 
Participantes no contexto a ser estudado e a coleta das informações

Como modelo de organização da investigação, optou-se por abordar os participantes enquanto estivessem prestanto cuidados no serviço do bloco operatório central de um Hospital Público das ilhas de Sotavento, localizado na Cidade da Praia, em Cabo-Verde.

O bloco operatório realiza 3.317 cirurgias por ano, sendo 2.035 intervenções cirúrgicas electivas de todas as especialidades e 1.282 intervenções de urgências, também de todas as especialidades cirúrgicas, de acordo com o Relatório Estatístico do Ministério da Saúde da República de Cabo-verde (Cabo Verde, 2013).

Este serviço conta com uma equipe multidisciplinar, composta por cirurgiões, anestesistas, enfermeiros e agentes dos serviços gerais, com cobertura nos turnos diurno e noturno.

O período de coleta foi desenvolvido no intervalo temporal de abril a junho de 2015, contemplando as observações participantes e entrevistas semiestruturadas.

A observação foi do tipo participante, sem intervenção nas ações (Spradley, 1980) da equipe do bloco operatório. Não foi ponderada a quantidade de intervenções, mas a diversidade de singularidades que pudessem ser observadas e a intencionalidade para o foco do estudo, em uma compreensão dos significados atribuídos dentro do contexto social que permitissem a descoberta de significativas, precisas e credíveis.

Após observação descritiva do contexto, foram selecionadas como dimensões para observação focalizada: (1) uso de escovas na lavagem cirúrgicas das mãos; (2) uso de espuma (clorexidina ou iodopovidona) para lavagem cirúrgica das mãos; (3) tempo da lavagem cirúrgica de 2 a $5 \mathrm{~min}$; (4) utilização de um che- cklist para cirurgias seguras; (5) certificação dos materiais esterilizados.

As observações focalizadas totalizaram 100 horas, com idas diárias de 2 horas, distribuídas pelos turnos diurno e noturno. Pôde-se considerar 50 momentos de observação.

A medida em que eram realizadas as observações e surguiam a necessidade de clarificar o que ocorria no cotidiano, foram sendo realizadas as entrevistas semiestruturadas $(\mathrm{E} 1, \ldots, \mathrm{E} 6)$, com vistas a maior clareza e magnitude do que se pretendia nesta fase de coleta de dados, tendo como pressupostos orientadores os questionamentos: (1) o que caracteriza a realidade sociocultural dos enfermeiros em bloco operatório? (2) Em que medida a vivência sobre a segurança do doente no bloco operatório remete à construção de uma identidade social específica do enfermeiro? (3) Quais características manifestam-se como as mais adequadas à segurança do doente nos processos sociais e na relação com os enfermeiros em contexto de bloco operatório?

Participaram das entrevistas seis enfermeiros, após consentimento livre e esclarecido, incluídos como participantes apenas os efetivos no quadro de serviço.

Seguiu-se o critério de amostragem teórica, à medida que as entrevistas mostravam saturação pelo esgotamento do conteúdo, pela apresentação das redundâncias e duplicidades de ideias (Morse, 1994).

\section{Análise dos Dados}

As observações foram agrupadas em três grandes dimensões e apresentando o número de entradas em cada combinação através de uma matriz quadrática de relação teórico-empírica (Tabela 1).

A interpretação das entrevistas teve como suporte o Qualitative Data Analysis Software 
(Nvivo, versão 10.0) e fundamentou-se nas técnicas de análise de conteúdo, propostas por Bardin (2011).

$\mathrm{Na}$ etapa de pré-análise, foram realizadas leituras repetitivas da constituição do corpus referente ao material das transcrições das entrevistas.

A exploração deste material preparou a codificação em categorias, a partir de recortes, classificação e agrupamento das unidades de registro e de contexto. Seleccionou-se como unidades de registro a palavra e a unidade de contexto, o parágrafo.

$\mathrm{Da}$ análise, emergiram cinco categorias explicativas e integradoras dos conceitos sobre segurança do doente no contexto do bloco operatório: (1) Gestão de conflitos; (2) Comunicação entre a equipe interdisciplinar; (3) A pessoa como centralidade dos cuidados em contexto cirúrgico; (4) Insegurança nos cuidados por escassez de normas e supervisão; (5) Insegurança nos cuidados relacionados com materiais esterilizados.

Para caracterizar a construção explicativa sobre a segurança do doente em contexto do bloco operatório, utilizaram-se enxertos dos discursos os mais paradigmáticos possíveis e representativos.

\section{Aspectos Éticos}

O estudo foi desenvolvido por um recorte do projecto percepção dos enfermeiros sobre a segurança do doente.

Os preceitos éticos e legais de pesquisa com seres humanos foram respeitados, após ter aprovação e autorização do Comitê Nacional de Ética em Pesquisa para a Saúde de Cabo-Verde, conforme aprovação n. ${ }^{\circ}$ 19/2015, ao artigo n. ${ }^{\circ}$ 11 do Decreto-lei n. ${ }^{\circ}$ 26/2007, de 30 de julho.

\section{Apresentação e discussão dos resultados}

As observações das práticas da equipe multiprofissional no bloco operatório foram categorizadas com base nas recomendações para segurança do doente em bloco operatório, preconizada pela Organização Mundial de Saúde (WHO, 2009). Evidenciaram-se três dimensões teóricas: (1) lavagem cirúrgica das mãos, com escovação de 2 a 5 minutos com solução de clorexidina, (2) utilização de checklist para cirurgias seguras; (3) verificação de indicadores de esterilização (Tabela 1 ).

\begin{tabular}{|lccc|}
\hline & \multicolumn{2}{c|}{ Observações } \\
\cline { 2 - 3 } Dimensões teóricas & $\mathbf{N}$ & Navorável & Não favorável \\
\cline { 2 - 3 } $\begin{array}{l}\text { Lavagem cirúrgicas das mãos com } \\
\text { escovação durante 2 a 5 min com solução } \\
\text { clorexidina }\end{array}$ & 10 & $\mathbf{N}$ \\
\hline $\begin{array}{l}\text { Utilização de checklist para cirurgias } \\
\text { seguras }\end{array}$ & 0 & 50 \\
\hline Verificação dos indicadores de esterilização & 14 & 36 \\
\hline
\end{tabular}

TABELA 1: Relação entre evidências para segurança dos doentes e a prática da equipe multiprofissional no bloco operatório: Matriz teórico-empírica. 
Os 50 momentos de observação representaram a identidade cultural do contexto em estudo e evidenciaram elevada e baixa relação, com base no número de ocorrências $(\mathrm{N})$, em combinações identificadas como: favorável e não favorável.

A identidade sobre aspectos básicos do bloco operatório evidentes na relação com os processos sociais cotidianos constrói-se em maior número em observações não favoráveis para a segurança do doente.

Os resultados das observações quantificadas foram explicados através das entrevistas realizadas. Para análise inicial do corpus, foi realizado agrupamento por semelhança lexical, representado em dendograma (Figura 01), com soluções gráficas para três cluster como a melhor solução explicativa.

Isto significa que as cores iguais apresentam agrupamentos de palavras ou códigos estruturados que se repetem com frequência nas fontes e utilizam-se mais vezes as mesmas palavras no recurso lexical. Esta análise inicial ajuda a identificar diferentes padrões ideológicos que existem nos textos, ou seja, os trechos mais representativos dos discursos.

As palavras com mais contribuição por frequência ( $\mathrm{CPF}$ ) relacionam-se com um modelo empírico que na perspectiva dos enfermeiros explica que para a segurança $(\mathrm{CPF}=21)$ dos cuidados $(\mathrm{CPF}=18)$ no bloco $(\mathrm{CPF}=15)$ operatório $(\mathrm{CPF}=13)$ deve-se ter como centralidade o doente $(\mathrm{CPF}=30)$ em condição cirúrgica.

$\mathrm{O}$ cuidado descreve-se na lógica de uma transição e adaptação tanto na perspectiva de superação das demandas do cliente quanto na adequação aos cuidados prestados pelo enfermeiro (Lopes, 2005). Mesmo com a importância da complexidade de tecnologias de modernas, nos cuidados, é de relevância que a pessoa não deve ser vista pelo enfermeiro como um ser que necessita de um reparo, um sujeito doente (Avanci, Furegato, Scatena, \& Pedrão, 2009).

Por similaridade de palavras, pode-se compreender que as representações ideológicas se formam em função de normas $(\mathrm{CPF}=11)$, as quais são necessárias para uma comunicação eficaz $(\mathrm{CPF}=12)$ e com isto melhorar $(\mathrm{CPF}=19)$ as práticas $(\mathrm{CPF}=15)$ no contexto.

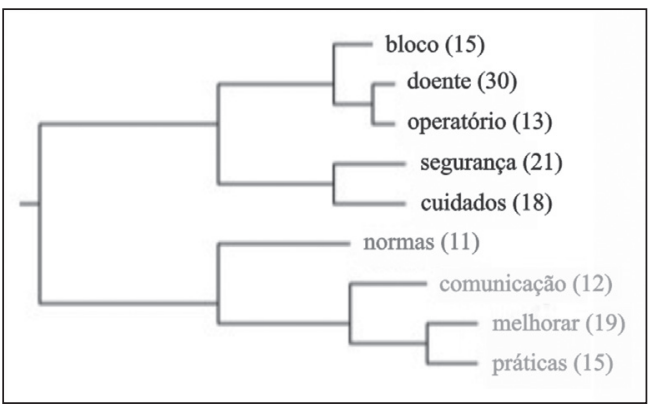

Figura 01: Representação da frequência de palavras similares, distribuídas em três cluster.

A comunicação não é de forma unilateral, deve-se exercer o ato de ouvir, não com um julgamento prévio que tenha como base as próprias convicções, assim como deve se fazer entender, considerando os aspectos socioculturais que envolvem essa relação (Santos \& Sawaia, 2000). Nesta lógica, a comunicação constitui-se como um instrumento de cuidado no contexto hospitalar, fazendo parte da assistência prestada pelo enfermeiro, renomeando-se, então, como uma comunicação terapêutica, considerando as emoções desse cliente, trabalhando, assim, ansiedade e dúvidas, diminuindo o estresse e aliviando expectativas (Avanci, Furegato, Scatena, \& Pedrão, 2009).

Essa comunicação terapêutica se faz necessária em situações em que os indivíduos estão vivenciando uma transição. Considerando a vivência dos doentes em bloco cirugico como 
uma crise ou transição, a informação assume um importante papel na reorganização que permita ultrapassá-las. Deve-se ponderar que apenas a informação não permite a transição, mas como o autor refere essa informação quando solicitada pelo cliente ou família, de acordo a suas necessidades, facilitando a reorganização. Os componentes do cuidado como compreensão, toque, dedicação, calor humano, doação, compaixão, desvelo, comunicação são importantes aliados diante de uma transição exacerbada (Camillo, Nóbrega, \& Theo, 2010).

\section{SEGURANÇA DO DOENTE EM CONTEX-} TO DO BLOCO OPERATÓRIO:

\section{CONSTRUÇÃO EXPLICATIVA}

\section{Gestão de conflitos}

Ao pensar nas competências do líder na gestão dos conflitos, para Salvagni \& Viau (2014), é necessário saber ouvir e ser ouvido pelos outros, aceitar elogios, críticas e sugestões. No entanto, uma das dificuldades identificadas nos discursos dos enfermeiros investigados esteve relacionada às competências da liderança.

“As boas práticas para segurança do doente no bloco está relacionado a uma boa comunicação, ter bom líder e ainda com as competências das chefias em saber gerir os conflitos e melhorar a comunicação".(E6)

A liderança tem sido objeto de vários estudos, com diversas definições e teorias. Cada autor apresenta o seu ponto de vista sobre o conceito de liderança, com intuito de apresentar um conceito contemporâneo e que relacione algumas variáveis, particularmente as relações entre o líder e os seus seguidores. Estes pontos devem ser vistos como oportunidades para crescer para um grande líder, sendo a comunicação um aspecto primordial a ressalvar.

O bloco operatório, devido ao alto nível tecnológico que apresenta e à especificidade de funções que exige, é um local privilegiado ao aparecimento de momentos de confusão, estresse, tensões emocionais e relacionais. Face à complexidade organizacional do bloco operatório é comum que o trabalho em equipe e a comunicação seja sistematicamente referido como fator humano fundamental para a qualidade do cuidado prestado neste setor (Santos \& Ferrão, 2015).

\section{Comunicação entre a equipe interdisciplinar}

De acordo a OMS (WHO, 2009), tal como acontece em outros sistemas complexos, a comunicação é essencial para o funcionamento seguro e eficaz da equipe cirúrgica.

A ausência da comunicação foi referida pelos enfermeiros investigados, quando afirmaram que ainda precisavam melhorar a comunicação no sentido de evitar o erro. Alegaram carência de comunicação com o doente e entre a equipe. $\mathrm{O}$ pensamento construído pelos entrevistados corrobora com Santos, Grilo, Andrade, Guimarães, \& Gomes (2010) quando referiram que a comunicação efetiva é vista na equipa multidisciplinar como algo muito importante, cuja finalidade é evitar o erro.

A comunicação torna-se difícil dentro das equipes de saúde interdisciplinares, nas quais a hierarquia resultante de uma cultura profissional e institucional leva alguns grupos profissionais e pessoas a manifesteram comportamentos neutros, de modo a sentirem dificuldades em expressarem-se por meio de um diálogo aceitável com outros profissionais.

"Apesar de ter uma percepção de que os cuidados aqui são seguros, penso que ainda precisamos melhorar a comunicação no bloco operatório, no sentido de evitar o erro." (E1).

"Os doentes ainda têm falta de informações sobre a doença” (E2). 
A Joint Comission for the Accreditation of Hospital Organizations formalizou um protocolo para reduzir a prevalência da "Cirurgia no Lado Errado". Neste, incluiu-se que processo de comunicação no bloco deveria assumir como atributos definidores a identificação do doente, o tipo, o lado e o nível da cirurgia. Tudo seguido da marcação, com tinta estável, do local da operação prevista. O chamado time-out é um verdadeiro briefing entre os membros da equipe, em que todo o procedimento é prospectivamente introduzido e revisitado (Fragata, 2010).

Considera-se a comunicação é um instrumento básico na prestação dos cuidados, em múltiplas dimensões de acção, contemplando orientação, informação assertiva e apoio, com a finalidade de atender às necessidades mais diversas e singulares do doente, família e da equipe multidisciplinar (Santos \& Ferrão, 2015).

A comunicação deve ser intencional, considerando os aspectos éticos e sendo compreendida por todos os envolvidos. A comunicação é parte essencial no processo terapêutico, esta envolve escutar cuidadosamente e interpretar inteligentemente. $\mathrm{O}$ enfermeiro deve considerar a comunicação com o doente e a equipe como um processo recíproco (Nakata \& Silva, 2005).

Em síntese teórica, a comunicação quando não ocorre de forma eficaz, em que os sujeitos participantes não estão plenamente envolvidos, ou quando essa comunicação é unilateral e não possui um conjunto de informações suficientes, que sejam esclarecedoras, pode tornar-se uma barreira que dificulta o enfrentamento e o processo de transição saudável (Santos \& Sawaia, 2000).

A pessoa como centralidade dos cuidados em contexto cirúrgico

Esta categoria temática descreve os sentimentos, em que se indagou-se preocupação dos enfermeiros no tocante à a pessoa, principalmente ao idoso.

Para Avanci, Furegato, Scatena \& Pedrão (2009), a relação entre enfermeiro e cliente é essencial no ato de cuidar, pois é a partir dela que os vínculos são estabelecidos, a confiança mútua é construída (e passa a ser uma mais valia nesse processo) e os planos de cuidados deixam de estar centrados apenas em ações curativas, mas envolvem a participação do sujeito como agente ativo, levando-se em consideração vivências e experiências deste. O relacionamento enfermeiro-cliente facilita a saúde, fazendo com que o cliente, através da experiência que vivencia, restaure a harmonia interior e o potencial de cura (Chistóforo, Zagonel, \& Carvalho (2006).

Os enfermeiros pesquisados reconhecerem a importância de centrar o cuidado no doente e identificaram esta necessidade como uma falha no processo de cuidar. Este aspecto corrobora com Monteiro (2014), ao referir que as falhas no relacionamento interpessoal no binômio enfermeiro-doente está na necessidade de ouvir os pacientes, ter mais leituras sobre o assunto e planejar os cuidados, ponderando o cuidado holístico, dinâmico e flexível.

"Há falta de atenção á pessoa idosa."(E1)

"Ter pessoas preparadas para dar as informações aos doentes durante 24 horas, dar ênfase a comunicação e redobrar os cuidados à pessoa idosa."(E3)

A complexidade da saúde está na capacidade de entender as partes mais simples para compreender o todo. Para Monteiro (2014), o enfermeiro do perioperatório é responsável e responsabilizado por todas as atividades relacionadas com o cuidar, uma vez que utiliza conhecimentos, capacidades e habilidades adquiridas para apoiar psicologicamente o doente, a família e outras pessoas significativas, pois deverá garantir a segurança, o con- 
forto e um profundo conhecimento do meio envolvente.

Desde então, a enfermagem de bloco operatório abandonou a tradicional intervenção, predominantemente técnica, para adoptar, progressivamente, uma abordagem holística da pessoa doente na satisfação das suas necessidades, tendo a qualidade dos cuidados melhorado muito, nas últimas décadas, desde o momento que centrou a atuação no doente. A enfermagem desenvolveu, assim, um novo papel associado ao cuidar da pessoa com necessidade de cuidados cirúrgicos e anestésicos: a Enfermagem Perioperatória (Silva, 2006).

$\mathrm{O}$ cuidado não se limita à realização de uma tarefa ou um procedimento de assistência clínico, curativo e hospitalar, reconhecem a importância de a enfermagem deter um conhecimento técnico-cientifico articulado com uma visão humanística (Avanci, Furegato, Scatena, \& Pedrão, 2009), pautado na diversidade de saberes tanto atitudinal, de habilidade, como cognitivo. A partir deste olhar, o cuidado rompe barreiras e existe quando é compreendido e aceito pelos seus atores: cliente, família e enfermeiro, colocando como foco o cliente e considerando a história de vida, experiências, valores e contextos.

Insegurança nos cuidados por falta de normas e supervisão

Para garantir a segurança do doente no bloco operatório, foi referido como essencial a implementação de normas e protocolos necessários para práticas seguras.

Nos discursos, também foram referidos sentimentos negativos relacionados à sobrecarga e dimensão do trabalho e, também, à impotência diante de determinadas situações, como a carência de materiais e equipamentos de qualidade.

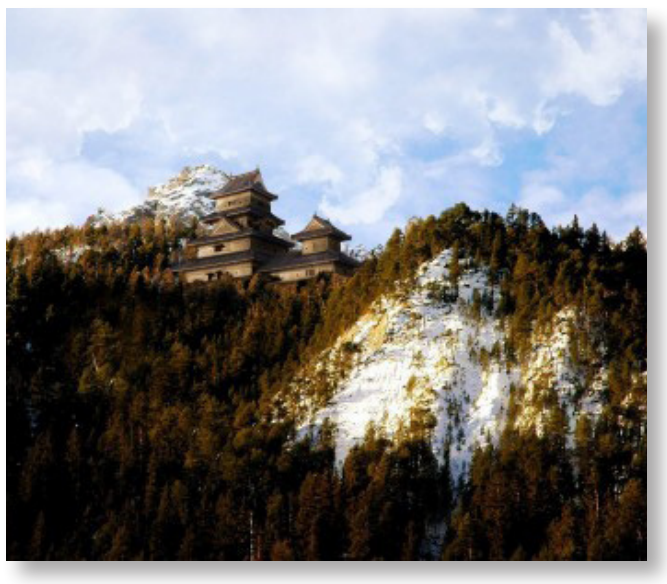

"Adoptar normas com a introdução de um checklist". (E4)

"A segurança doente está comprometida no bloco operatório por falta de materiais, como EPI, roupas esterilizadas que não dão segurança, o sistema de climatização do bloco é deficitário, há falta de cobertores para aquecer os doentes, há falta de pessoas especializadas para cuidar dos doentes".(E2)

A prevenção das infecções associadas aos cuidados da saúde deve constituir um dos componentes críticos de qualquer programa de segurança. Para o controle e a implementação de medidas de prevenção, é essencial a identificação dos fatores de risco. Estas medidas devem começar com a concepção e o funcionamento do bloco operatório, abrangendo o controle ambiental e as boas práticas para a prevenção da infecção. A prevenção da infecção do local cirúrgico passa pelos cuidados, com a utilização dos materiais e o tratamento de dispositivos médicos, pela assepsia e pelo comportamento da equipe (Ercole, Chianca, Duarte, Starling, \& Carneiro, 2011).

Insegurança nos cuidados devido à carência de normas e supervisão

Os entrevistados apresentaram discurso defensável sobre a importância de considerar 
práticas seguras no bloco operatório. Em termos gerais, a segurança do doente é definida por todas as práticas diárias, para que os cuidados no bloco operatório não sejam inseguros, na medida em que não existe um controle rigoroso de esterilização. Com isso, enfatizaram nesta categoria a segurança quanto à roupa cirúrgica e a outros materiais esterilizados.

"Vigiar o serviço de esterilização, criação de normas de procedimentos, superfícies, melhorar a comunicação entre a equipe e adoção de normas e protocolos onde toda a gente fale a mesma linguagem"(E3)

"As boas praticas no bloco operatório estão relacionadas com uma boa esterilização dos matérias".(E5)

Os enfermeiros do bloco operatório demonstraram preocupação com a segurança do doente quando realçaram a insegurança nos cuidados relacionados com os materiais esterilizados e o uso do formaldeído em forma de comprimidos para desinfecção e esterilização dos materiais, assumindo-se a existência do risco de infecção aos doentes.

"Insegurança nos cuidados, materiais esterilizados de forma insegura, como as roupas, os ferrosos cirúrgicos e entre outros, existe um risco de infecção cruzada entre os doentes”.(E3)

"Uso do formol em comprimidos para esterilização, prática de desinfecção incorrecta. Prática de esterilização dos materiais ou seja soluções incorretas".(E6)

Algumas infecções hospitalares são evitáveis e outras não. Infecções preveníveis são aquelas em que se pode interferir na cadeia de transmissão dos micro-organismos. A interrupção dessa cadeia pode ser realizada por meio de medidas reconhecidamente eficazes, como a lavagem das mãos, o processamento dos artigos e superfícies, a utilização dos equipamentos de proteção individual, no caso do risco laboral, e a observação das medidas de assepsia. Infeções não preveníveis são aquelas que ocorrem a despeito de todas as precauções adotadas, como se pode constatar em pacientes imunologicamente comprometidos, e são originárias da microbiota (Pereira, Ribeiro, Mendonça, Tipple, Souza, Palos, \& Barreto, 2011).

A competência do enfermeiro, além de estar direcionada para as práticas organizacionais, estar associada á relação destas práticas com a segurança do doente. Nesta perspectiva, os profissionais da saúde, preocupados com o ser humano, ao prestar o cuidado ao doente que será submetido a uma cirurgia, devem desenvolver habilidades de alta competência técnica e elevado conhecimento científico (Chistóforo, Zagonel, \& Carvalho, 2006).

\section{CONSIDERAÇÕES FINAIS}

A problemática descreve potencialidades no modelo cuidar que pouco considerando as dificuldades em que se desenvolvem estratégias para um cuidado interativo, com vistas aos problemas relativos às definições de política pública, organização do processo de trabalho e indicadores de qualidade dos serviços avaliados em múltiplas perspectiva.

Conhecer a percepção de enfermeiros sobre a segurança do doente é um desafio que permite o reconhecimento e a aproximação de vivências pouco exploradas em contexto do bloco operatório. Discorrer com enfoque na segurança do doente é parte prioritária para assimilação e aplicação desses conhecimentos e identificação dos resultados eficazes na prática.

A segurança do doente percepcionado por enfermeiros assenta, em alguns aspectos fundamentais, como a comunicação para gestão dos conflitos, a pessoa como centralidade dos 
cuidados em contexto cirúrgico, os cuidados inseguros relacionados ao déficit de matérias esterilizados e ausência de normas, auditorias e supervisão.

$\mathrm{O}$ cuidado de enfermagem mostra-se como um um momento de interação. Tanto de auto-interação, reconhecendo-se a si como enfermeiro que cuida, e a partir daí interpretando, checando, observando, analisando, refletindo para realização de ações e interações possuidores de significado e significante tanto para os enfermeiros como para o doente.

Evidenciou-se a necessidade de comunicação eficaz que envolva os sujeitos participantes do processo de cuidar, na perspectiva de que a pessoa como centro do cuidado é possuidora de visões próprias de mundo e respondem de forma plural aos estímulos, com indicadores de assertividade condutora de um cuidado congruente.

A segurança do doente apresenta importante indicador para os profissionais do bloco operatório, juntamente com a instituição, sendo que a ausência de normas, protocolos, supervisão e auditorias constituem pontos críticos para segurança do doente no contexto do bloco operatório.

\section{REFERÊNCIAS}

- Alpendre, F. T. (2014). Cirurgia segura: validação de checklist pré e pós-operatório. (Mestrado), UFPR, Curitiba.

- Avanci, R. C., Furegato, A. R. F., Scatena, M. C. M., \& Pedrão, L. J. (2009). Relação de ajuda enfermeiro-paciente pós-tentativa de suicídio. Revista Eletrônica Saúde Mental, Álcool e Drogas, 5(1), 65-78. Acessado em 15 de novembro de 2012, disponível em: http://www2.eerp.usp. br/resmad/artigos.asp

- Bardin, L. (2011). Análise de Conteúdo. Lisboa: Edições 70.

- Cabo Verde, Ministério da Saúde. (2013). Relatório Estatístico 2013. República de Cabo Verde: Ministério da Saúde.

- Cambotas, C. M. J. C. (2015). A prática baseada na evi- dência em contexto da enfermagem peri operatória. (Mestrado), Instituto Politécnico de Setúbal, Portugal.

- Camillo, S. O., Nóbrega, M. P. S. S., \& Theo, N. C. (2010). Percepções de graduandos de enfermagem sobre o ato de ouvir na prática assistencial. Revista da Escola de Enfermagem da USP, 44(1), 99-106.

- Chistóforo, B. E. B., Zagonel, I. P. S., \& Carvalho, D. S. (2006). Relacionamento enfermeiro-paciente no pré-operatório: uma reflexão à luz da Teoria de Joyce Travelbee. Cogitare Enfermagem, 11(1), 55-60.

- Corbin, J., \& Strauss, A. (2008). Basics of Qualitative Research: Techniques and Procedures for Developing Grounded Theory (3 ${ }^{\text {rd }}$ ed.). Thousand Oaks, CA: Sage.

- Duarte, S. D. C. M., Stipp, M. A. C., Silva, M. M. D., \& Oliveira, F. T. D. (2015). Adverse events and safety in nursing care. Revista Brasileira de Enfermagem, 68(1), 144-154.

- Ercole, F. F., Chianca, T. C. M., Duarte, D., Starling, C. E. F., \& Carneiro, M. (2011). Surgical site infection in patients submitted to orthopedic surgery: the NNIS risk index and risk prediction. Revista Latino-Americana de Enfermagem, 19(2), 269-276.

- Fragata, J. I. (2010). Erros e acidentes no bloco operatório: revisão do estado da arte. Revista Portuguesa de Saúde Pública, Supl(10), 17-26.

- Gebrim, C. F. L., Melchior, L. M. R., Amaral, N. M., Barreto, R. A. S. S., \& Palos, M. A. P. (2014). Tricotomía preoperatoria: aspectos relacionados con la seguridad del pacientes. Enfermería Global, 34(13), 252-263.

- Lopes, M. J. (2005). Os clientes e os enfermeiros: construção de uma relação. Revista da Escola de Enfermagem da USP, 39(2), 220-228.

- Monteiro, M. O. M. F. (2014). Necessidades de Informação dos Familiares/Pessoa Significativa dos Clientes Cirúrgicos, no Bloco Operatório. (Mestrado), Instituto Politécnico de Setúbal, Portugal.

- Morse, J. M. (1994). Aspectos essenciais de Metodologia de Investigação Qualitativa. Portugal: Formasau.

- Nakata, S., \& Silva, W. V. (2005). Comunicação: uma necessidade percebida no período pré-operatório de pacientes cirúrgicos. Revista Brasileira de Enfermagem, 58(6), 673-676.

- Paranaguá, T. T. D. B., Braga, Q. D. P., Bezerra, A. L. Q., Camargo-Silva, A. E. B., Azevedo-Filho, F. M. D., \& Sousa, M. R. G. (2014). Eventos adversos: instrumento de gerencia de la asistencia para la seguridad del paciente en el servicio de urgencias. Enfermería Global, 34(13), 206-218. 
- Pereira, M. D. D., Souza, D. F., \& Ferraz, F. (2014). Segurança do paciente nas acções de enfermagem hospitalar: uma revisão integrativa de literatura. Revista Inova Saúde, 3(2), 55-87.

- Pereira, M. S., Ribeiro, L. C. M., Mendonça, K. M., Tipple, A. C. F. V., Souza, A. C. S., Palos, M. A. P., \& Barreto, R. A. S. S. (2011). Grupo de pesquisa em enfermagem na prevenção e controle de infecções: 20 anos de contribuições. Revista Eletrônica de Enfermagem, 13(1), 124129. Acessado en 07 de maio de 2015, disponível em: http://www.fen.ufg.br/revista/v13/n1/v13n1a14.htm.

- Rodrigues, F. R. A. (2015). Interdisciplinaridade como estratégia para um cuidado de saúde culturalmente competente em serviço de infeciologia. Revista de Estudos Interculturais, 3(3), 1-17.

- Salvagni, J., \& Viau, M. R. (2014). O papel do lider na administração de conflitos. Revista de Administração da Fatea, 9(9), 121-131.

- Santos, A. P. L., \& Ferrão, S. A. D. S. (2015). Comunicação
Efectiva na Transferência da Pessoa em Situação Critica: revisão de literatura. (Mestrado), Escola Superior de Enfermagem de Lisboa, Portugal.

- Santos, M. C. D., Grilo, A., Andrade, G., Guimarães, T., \& Gomes, A. (2010). Comunicação em saúde e a segurança do doente: problemas e desafios. Revista Portuguesa de Saúde Pública, Supl(10), 47-57.

- Santos, V. L. C. G., \& Sawaia, B. B. (2000). A bolsa na medição "estar ostomizado”-“Estar Profissional”: análise de uma estratégia pedagógica. Revista Latino-Americana de Enfermagem, 8(3), 40-50.

- Silva, M. F. (2006). Acolhimento do doente no Bloco Operatório. Nursing, 16(208), 36-41.

- Spradley, J. P. (1980). Participant Observation. Florida: Holt.

- WHO, World Health Organization. (2009). The Research Priority Setting Working Group of the World Alliance for Patient Safety. Geneva: World Health Organization.

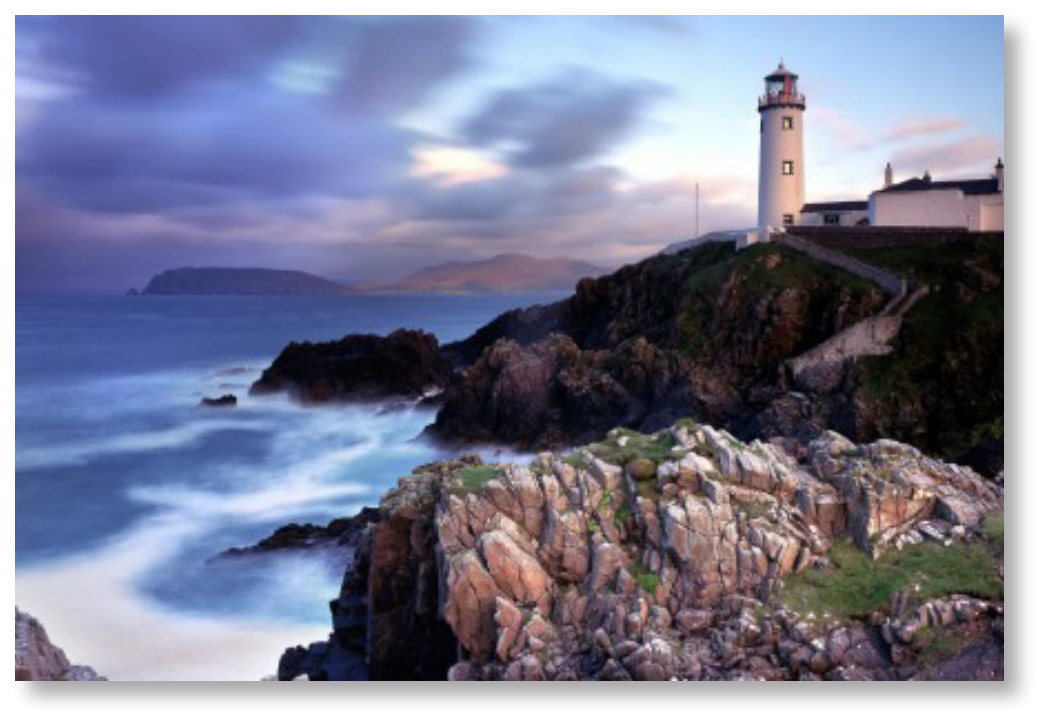

